\title{
ADVANCES OF TRANSMISSION LINE KICKER MAGNETS
}

\author{
L. Ducimetière, CERN, Geneva, Switzerland
}

\section{Abstract}

Fast pulsed magnets or kickers are widely used in circular accelerators for injection, fast extraction and beam excitation. As from the early 60 's transmission line type kicker magnets have been employed to produce rectangular field pulses with good rise time. Over some 40 years this technology has evolved with the rising requirements. While the necessary kick strength has increased with the particle beam energy the strive for efficiency has pushed developments towards lower impedance systems and/or short circuited magnets. The flat top ripple is constrained by the maximally tolerable beam oscillation. The beam intensity can impose a screening of the magnet yoke. The most advanced features implemented in recent transmission line kicker magnets are reviewed and illustrated with examples from different laboratories.

\section{INTRODUCTION}

In circular accelerators, fast injection and extraction of beam is generally performed using kicker magnets. These fast pulsed electromagnets are aligned with the circulating beam and kick the beam only once. Distinction between the bunches to be injected/extracted and the circulating beam is ensured by fast rise and fall times of the magnetic field, typically in the order of tens to hundreds of nanoseconds. Thus, the rise, respectively fall time is shorter than the distance between trains of bunches and, except for one- or multi-turn beam extraction, the pulse is shorter than one turn. The price to pay for this speed is a relatively low field strength. For this reason, kicker magnets are generally combined with electromagnetic septa, which complete the injection/extraction by deflecting the beam further.

The main specifications for kicker magnets are derived from the bunch energy, length, and spacing, from the tolerable beam oscillation and from the available space. Because of coupling effects, the beam intensity can also largely influence the design. The technological evolution has essentially followed the requirements of accelerators.

\section{DESCRIPTION OF TRANSMISSION LINE KICKER MAGNETS}

Electromagnets exhibit a lumped inductance which limits the rate of rise/fall of the current. With $\mathrm{L}_{m}$ the magnet inductance, $L_{s}$ the stray inductance and $Z$ the impedance of the pulse generator, the rise time is exponential with a time constant $t_{c l}$ of

$$
t_{c l}=\left(L_{m}+L_{s}\right) / Z
$$

The 0 to $95 \%$ rise time $t_{r}$ is as long as $t_{r}=5 t_{c l}$.

To overcome this difficulty, the first transmission line kicker magnet has been developed at CERN in the early 60's. The magnet is split in several $(n)$ cells, each connected to ground with decoupling capacitors $C_{n}$. If $L_{n}$ is the cell inductance,

$$
C_{n}=L_{n} / Z^{2}
$$

The characteristic impedance $Z$ is matched to the impedance of the generator. The magnet is generally terminated by its own impedance. The current pulse transits through the magnet with a delay $t_{m}$, the so called filling time:

$$
t_{m}=n \sqrt{L_{n} C_{n}}
$$

The field rise time $t_{r}$ starts with the beginning of the current pulse at the magnet entrance and ends with the end of the same pulse at the exit of the magnet (Fig. 1). It can then be strictly defined as the sum of the current rise time and the magnet filling time $t_{m}$.

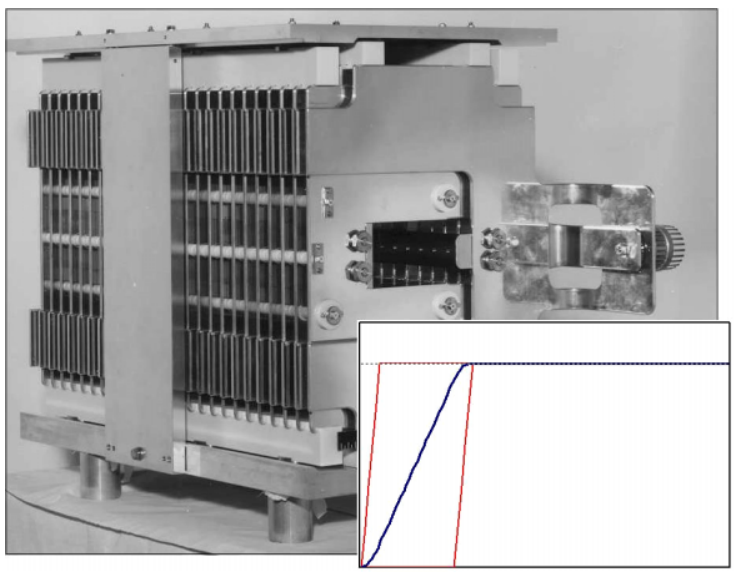

Figure 1: Transmission line kicker magnet and corresponding field rise time vs time.

Therefore, transmission line kicker magnets perform much faster than equivalent lumped magnets. As trade off, design, construction and operation are more complicated and costly. The first generation was housed in a vacuum tank and used parallel metallic plates as decoupling capacitors [1]. Depending on the application, magnets have evolved into different directions. This will be looked at in more detail in the following chapter.

\section{EVOLUTION OF THE TECHNOLOGY}

\section{Optimising the Rise Time}

The leading edge of the exciting current contributes to the field rise time and should thus be optimised. Pulse Forming Lines (PFLs) or Pulse Forming Networks (PFNs) associated with speeding front cell(s) are used for this purpose. In spite of the latest progress with solid-state devices, deuterium thyratrons in coaxial housings remain to date the fastest commercially available switches, either in single or multi-gap configuration. Recent 4.5 " diameter 
tubes [2] are preferred for low impedance systems and long pulses because of their peak current, total conducted charge capabilities and smaller stray inductance. Switching characteristics are optimised with a fast and powerful triggering of grid 2 jointly with about $1 \mu$ s prepulsing on grid 1 . Switching speed is further improved by connecting saturable inductors in series [3]. Such an increase of the current rate of rise also reduces the commutation losses of the thyratrons [4]. Multi-gap thyratrons produce displacement currents, generated by the capacitive load of the gaps which collapse successively before the main discharge. Systems with short magnets and relatively high impedance are sensitive to this current, which contributes to the start of the field before the main rising edge. Studies carried out at TRIUMF show that saturable inductors are also very efficient to suppress the displacement current [5] and suggest installing them as close as possible to the magnet input. As example, CERN has used this technique for the upgrade of the PS injection and Booster recombination kickers KFA10 [6, 7], ANL for the IPNS extraction kicker [8] and BNL for the SNS extraction systems [9]. The delay depends of the voltage. Possible reflections reaching the magnets after the pulse are limitative.

Because the field builds up until the end of the current rise at the output of the magnet, it is important that the pulse does not degrade while travelling through the magnet. Thus the magnet cut-off frequency is a key parameter, especially with field rise times below some $100 \mathrm{~ns}$. With $L_{c s}$ being the stray inductance of the cell capacitance, the cut-off frequency $f_{c}$ is given by:

$$
f_{c}=\frac{1}{\pi \sqrt{\left(L_{n}+4 L_{c s}\right) \times C_{n}}}
$$

The above equation shows the importance of small cell size and low stray inductance. $L_{c s}$ takes account of the leakage inductance of decoupling capacitors and the negative forward magnetic coupling between the cells. Interleaved high voltage plates as cell capacitance provide the lowest possible leakage inductance and also serve as cell boundary to minimise the coupling. They shall extend as much as possible from the ferrite core.

Figure 2: Magnet cell from the FNAL Tevatron injection kicker magnet showing the coupling loops.

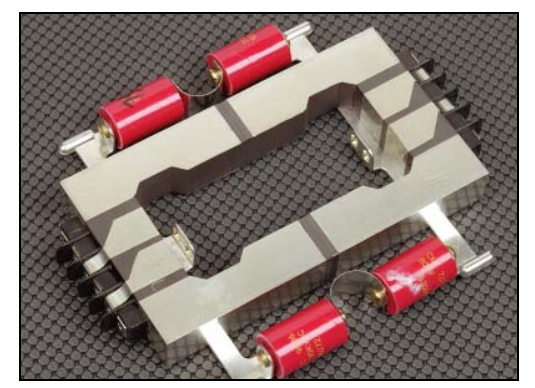

Furthermore, artificial counter-coupling of adjacent cells has been tried at CERN as early as 1979 [10], but remained at the prototyping state, mainly because of implementation difficulties in the surrounding high voltage environment. It consists of a single turn stripe counter-wound between adjacent cells. It pre-injects in the non-excited cell a magnetic flux opposite to the negative coupling effect. This effect has been further described and modelled at TRIUMF in 1989 [11]. More recently, counter-coupling has been successfully applied at FNAL for the Tevatron injection kicker magnets [12] (Fig. 2). Building the magnet for operating in an inert liquid dielectric has led to a reliable application.

To gain in filling time by a factor of two compared to a classical C-topology FNAL and KEK used a windowframe topology [12, 13] (Fig. 2). It can be considered as two symmetrical C-magnets energised independently with opposite polarities, the so called "balance drive". As trade off it requires two generators. This topology is also employed to limit beam coupling.

\section{Increasing the Kick Strength}

With increasing accelerator energy magnets needed to become stronger. Because the voltage is limited by the technology, improvements in kick strength are based on increases in length and exciting current. In both cases, the filling time increases proportionally. Magnet lengths, typically of 0.2 to $0.5 \mathrm{~m}$ in accelerators of a few hundred meters circumference [1], exceed $2.7 \mathrm{~m}$ for the LHC ring of $27 \mathrm{~km}$ [14].

A straightforward way to double the current, thus the kick strength but also the filling time, is to use short circuited magnets, as for example for the CERN PS Booster Ejection and Recombination kickers [6] or the KEK PS extraction [13]. The wave reflected by the shorted end propagates back through cables to the generator which should be designed for systematic negative voltage excursions and finally for absorbing the pulse energy. This solution is not widely used because of likely reflections occurring during/after the pulse, due to small mismatch at the various transition points. It is however the most straightforward way to try to increase the kick strength of existing systems when the rise time increase can be tolerated.

Kicker magnets with vacuum plate decoupling capacitors have their lowest limit of characteristic impedance around $12 \Omega$. To further decrease the impedance, high permittivity dielectric materials and discrete capacitors have been implemented [15].

CERN's $10 \Omega$ SPS extraction and $2.1 \Omega$ beam dump kicker magnets, built in the late 70 's, have discrete decoupling capacitors outside the vacuum tank [16]. This requires one vacuum-tight high voltage feed-through per cell, which drastically limits the cell quantity and length. Furthermore the external capacitors, typically a seriesparallel arrangement of either ceramic or oil impregnated capacitors, and especially their connection are up to one order of magnitude more inductive than parallel plate capacitors. These two factors result in a reduced bandwidth, a relatively long rise time and significant ripple. 20 years later, ANL was first to exploit ceramic capacitors interleaved with ferrite sections under vacuum for the four-cell Advanced Photon Source Injection kicker magnet [17].

Several transmission line kicker magnets built around a vacuum ceramic chamber have recently been developed. 
In 1995, SLAC built the SLC damping ring kicker magnet with moulded alumina loaded epoxy as dielectric for the capacitors [18]. The plate geometry is optimised and cells cast individually. In the late nineties FNAL adopted silicon potted between parallel plates for the Main Injector proton injection [19]. In 2001 KEK built the kicker for its photon factory storage ring following a similar technique, using vulcanised silicon rubber, and cell-per-cell moulding [20]. A design with high permittivity dielectric blocks interleaved with ferrite cores has also been used at BNL in 1997 for the RHIC injection kicker [21]. A weakness in voltage holding along the surface of the block sides has been reported. Using the permittivity of the ferrite yoke to achieve the decoupling capacitors is controversial but successfully applied at BNL as upgrade of the RHIC Injection Kicker [22, 23].

Commercial ceramic capacitors are the key for more powerful transmission line kicker magnets. The FNAL Tevatron injection $12.5 \Omega$ kicker magnet consists of about 30 cells, each with four $80 \mathrm{pF}$ cylindrical capacitors in a series parallel configuration (Fig 2). The dielectric is strontium titanate $\left(4700 \mathrm{ppm} /{ }^{\circ} \mathrm{C}\right)$. Because the magnet is insulated with inert liquid, standard epoxy housed capacitors have been used [12]. The NuMi kicker magnet currently under development at FNAL reuses the same principle with 46 cells and about $1.14 \mathrm{nF}$ per cell. The magnet impedance is $10 \Omega$ [24].

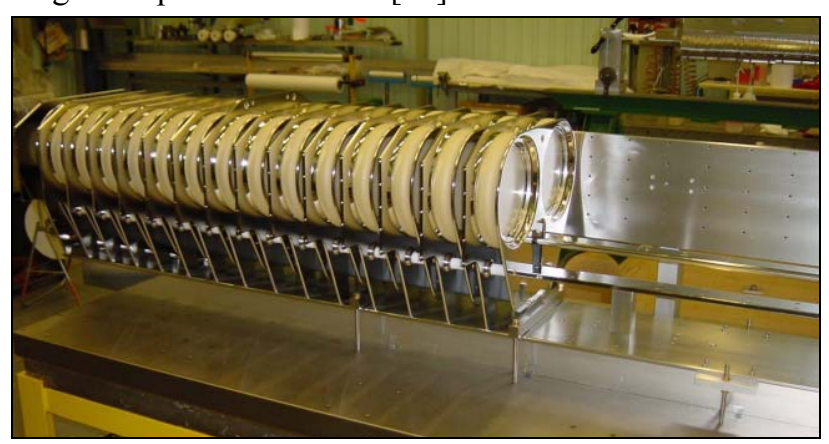

Figure 3: CERN LHC injection kicker magnet during assembly.

For its $5 \Omega$ impedance LHC injection kicker magnets, CERN retained a classical low inductance design with interleaving plates (Fig 3). The magnets works under high vacuum. Each of the 33 cells includes 2 ceramic plate capacitors with diameter $210 \mathrm{~mm}$ representing a capacitance of $4 \mathrm{nF}$. The capacitor dielectric is titanium dioxide $\left(750 \mathrm{ppm} /{ }^{\circ} \mathrm{C}\right)$. They are clamped in between the high voltage and ground plates and the connections are ensured by spring contacts at the level of the rims [14].

\section{Minimising the Ripple}

Acceptance for beam emittance blow-up of high energy accelerators is very small. Most frequent ripple sources are the ringing frequency of the magnet itself, various mismatches at transition points of the system and the generator. The use of magnets with many small cells and high cut-off frequency, of cable PFLs or small cell PFNs and careful matching of all elements of the system are proven solutions. PFN ripple is minimised with precise and continuous coils, good mutual coupling between cells and good stability of the capacitors. The grading of the capacitors compensates for conduction losses and ensures the flatness of long pulses [25]. Adjustment of the magnet impedance is obtained by accurate calculation $[14,26]$ or by variable spacing between conductors as foreseen in some of the FNAL kicker magnets [12,24].

With $\pm 0.5 \%$ ripple requirement, additional damping resistors have been added in the LHC injector kicker system, both in the PFNs and the magnets. In the PFNs, they are connected in parallel with the coils [25]. They are only active when reactive energy varies in the coils and do not affect the rise time as long as they are about one order of magnitude more resistive than the impedance. In the magnets, special UHV compatible resistors are connected in between the high voltage plates of adjacent cells [14]. They consist of two low inductance counterwound Kanthal ${ }^{\mathrm{TM}}$ wires on a ceramic rod. This attractive solution can generally be easily implemented on existing systems. It has also been used for the upgrade of the CERN SPS injection and extraction system [27]. For this latter, it was easier to connect dual damping resistors in series with the decoupling capacitors, outside the magnet. Series damping resistors were also used in the ANL Advanced Photon Source Injection kicker magnet [17].

\section{Beam Coupling Issues}

High intensity accelerators are very sensitive to resonances and the variation of longitudinal and transverse impedance. Kicker magnets, with their ferrite yoke, are disturbing elements in this respect. The beam coupling also induces heating of the ferrite yoke [28, 29], sometimes beyond the Curie temperature. In extreme cases it can affect the generator as well [30].

Resonances, usually at medium frequencies, can efficiently be eliminated by inserting transition pieces inside the tank to electrically connect the tank flange to the kicker module at each end $[31,32]$. When several magnet modules are installed in a common tank, the transition between modules is done in a similar way.

Impedance issues are addressed by screening the beam in the magnet gap. The screen should conduct the beam image current but at the same time not significantly attenuate the pulsed field. Ceramic chambers with uniform resistive coating grounded at both ends are widely used in lumped kicker magnets. As they are equivalent to a short-circuited secondary transformer winding with about $50 \%$ mutual coupling their total resistance should be large compared to the system impedance. This solution is rarely compatible with transmission line kicker magnets because of the fast rise time requirement. Moreover, at the cell scale, a uniform conductive layer offers paths for eddy-current loops.

In 1995, DESY has successfully introduced the concept of capacitively coupled stripes as screen in the DESY III dump kicker [29, 33]. It consists of two longitudinal interleaved combs made of sustained, regularly spaced metal stripes. Each comb is grounded at one end of the 
magnet. The stripes provide a path for the medium and high frequencies image current, while lower frequencies flow through the tank walls. The pulsed field can easily penetrate the free space between the stripes. The negative effect on the pulse is limited as only the coupling capacity between the two combs and capacity to ground are charged during the filling time. This concept is also used for the LHC injection kicker magnet [14]. Because the magnet length of over $2.7 \mathrm{~m}$, silver stripes are printed inside a $3 \mathrm{~m}$ long ceramic tube. The ceramic tube acts as support and in addition guarantees the insulation relative to the high voltage plates. The capacitive coupling is made at one end between the inside stripes and an outside grounded area. Therefore, it profits from the dielectric strength and permittivity of the ceramic. FNAL and KEK use an intermediate solution for the Tevatron and J-PARC vacuum ceramic chambers, respectively. Their inside is coated with a high resistivity layer and completed by metallic stripes on the outside. For FNAL, there are only two external stripes connected to the standard vacuum chambers of the accelerator via resistors or resistor/capacitor combinations [12, 24]. KEK has applied capacitively coupled stripes all around the chamber [34].

Figure 4: interleaved combs directly on the ferrite.

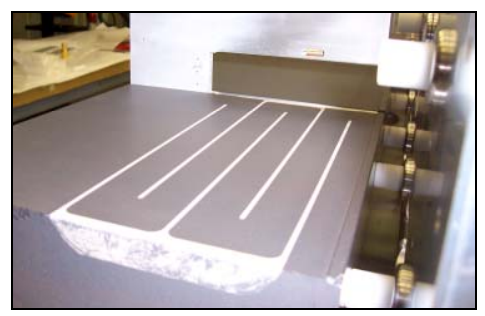

Such solutions cost up to $10 \mathrm{~mm}$ in aperture and therefore are rarely applicable to screen existing systems. Retrofit solutions are under study at CERN [35]. One promising option is to use interleaved silver combs at the level of each cell, directly printed on the ferrite by serigraphy and connected to the high voltage plates (Fig. 4). They do not reduce the aperture, and the high permittivity of the ferrite enhances the coupling. This solution is less attractive with magnets with short cells. Another alternative is to use the free space in the corners between the elliptical beam shape and the rectangular aperture to introduce ceramic inserts with suitable coating or stripes. In the window-frame topology, the symmetry axis is virtually at the ground potential and receives efficient screen plates.

\section{Design Tools}

Analogue simulation and finite element codes have helped circuit design for about 20 years. Model availability, manageable circuit size and computational power have followed the remarkable evolution of informatics. Simulations of circuits which include almost all known parasitic elements and non linearities are now possible. The optimisation process can be performed with an optimiser, starting from educated guesses of component values. It results in saving precious prototyping resources and further adjustment or correction. TRIUMF has made remarkable contributions in this domain, first within the CERN collaboration [25] and now within the BNL collaboration [36].

2D and 3D finite element codes now include transient analysis with eddy-current calculations. They are extremely effective tools to design fast pulsed magnets. For example the geometry of ferrites and conductors can be shaped to optimise the field homogeneity, the current distribution being computed by the code. The penetration of the pulsed field through a beam screen in a magnet aperture can also be accurately evaluated. AC analysis has also been used to assess the frequency dependency due to field effects for elements like PFN coils. From the results, equivalent frequency dependent models have been set up for analogue circuit simulation [25]. 3D analyses of connections and feedthroughs improve the impedance matching in order to avoid undesirable reflections [26].

\section{Measuring Fast Pulsed Magnetic Fields}

Static wire loops in the magnet aperture remain the preferred method. Careful design of probes includes shielding against the capacitive effect $[37,38]$. Because such measurements are not possible in the machine, capacitive pickups are sometimes implemented at both ends of the magnets. Both methods require integration of the collected signals, which is delicate with pulse flat-top lengths one order of magnitude longer than the rise time. The evolution from analogue to digital integration has enabled signal processing facilities [37]. During the flat top, the signal is very small and the offset is integrated over time. In order to optimise the accuracy, rise/fall times and flat-top periods are acquired at two different sensitivities and are rejoined after integration. Capacitive pickups do not allow an accurate measurement of the field when a beam screen is installed in the magnet aperture.

Alternative and original methods have also been used exceptionally, such as the variation of RF phase in a ferrite waveguide, presented by FNAL in 1997 [39], and the variation in polarization of a laser light in a crystal (Faraday Effect) by BNL [23].

\section{CONCLUSION AND OUTLOOKS}

Transmission line kicker magnets have been in constant evolution over the 40 years of their existence. The creativity of specialists all over the world has allowed to find adequate solutions for the many specific applications and keeps pushing the limits. Many factors which influence the rise time, kick strength and ripple have been explored, making extensive use of new design tools. After following the trend for ever larger circular accelerator, a most clean integration in high intensity machines is prominent among the future challenges.

\section{AKNOWLEDGEMENTS}

The author would like to thank Wu Zhang, Mike Barnes, Chris Jensen, Volker Mertens, Klaus-Dieter Metzmacher, and Luc Sermeus for there constructive discussions and support. 


\section{REFERENCES}

[1] D. Fiander, "A Review of the Kicker Magnet Systems of the PS complex", NPAC 1990, Moscow

[2] E2V Limited, http://e2vtechnologies.com/

[3] A. Brückner, "Kicking Protons, Fast and Cheap" PAC'71, Chicago.

[4] L. Ducimetière, D. Fiander, "Commutation Losses of a Multigap High Voltage Thyratron", Power Modulator Symposium, 1990, pp 248-253

[5] G.D. Wait, M.J. Barnes, K.D. Metzmacher, L, Sermeus, "The Application of Saturating Inductors for Improving the Performance of the CERN PS Kicker Systems", PAC'97

[6] K. Metzmacher, L. Sermeus, "The PSB Injection and Recombination Kicker Systems for LHC", CERNPS/CA/Note 2000-004.

[7] K.-D. Metzmacher, L. Sermeus, "The PS Injection Kicker KFA45 Performance for LHC", CERNPS/PO/Note 2002-015 (Tech.)

[8] L.I. Donley, J.C. Dooling, G.E. Mc Michael, "Using Ferrite as a Fast Switch for Improving Rise Time of IPNS Extraction Kicker' PAC'03.

[9] W. Zhang et al. "Design, Development, and Construction of SNS Extraction Fast Kicker System" PAC'03.

[10] G. Nassibian, "Travelling Wave Kicker Magnets with Sharp Rise and Less Overshoot", PAC'79.

[11] M.J. Barnes, G.D. Wait, R. Barnes, "Estimation of Negative Mutual Coupling between Adjacent Cells of a Magnetic Kicker", TRIUMF TRI-DN-89-K87

[12]C. Jensen, B, Hanna, R. Reilly, "A Fast Injection Kicker Magnet for the Tevatron", PAC'01, Chicago

[13]T. Kawakubo, E. Nakamura, S. Murasugi, "Low Beam-Coupling Impedance Kicker Magnet System Generating a High Magnetic Field with a Fast Rise Time" EPAC'2000.

[14] L. Ducimetière, N. Garrel, M. Barnes, G. Wait, "The LHC Injection Kicker Magnet", PAC'03

[15] W. Zhang, J. Sandberg, J. Tuozzolo, R. Cassel, L. Ducimetière, C. Jensen, M. Barnes, G. Wait, J. Wang, "An Overview of High Voltage Dielectric Material for Travelling Wave Kicker Magnet Application", Pulsed Power Conference 2002.

[16]P.E. Faugeras, E. Frick, C.G. Harrison, H. Kuhn, V. Rodel, G.H. Schröder, J.P. Zanasco, "The SPS Fast pulsed magnet systems", Modulator Symposium 76.

[17] J. Wang, "Design and Test Results of Kicker Units for the Positron Accumulator Ring at the APS", PAC'95

[18] T.S. Mattison, R.L. Cassel, A.R. Donaldson, G. Gross, "Fast and Reliable Kicker Magnets for the SLC Damping Rings", PAC'95, pp. 1915 -1917

[19]D. Qunell, C. Jensen, D. Tinsley, "Kicker System For $8 \mathrm{GeV}$ Proton Injection", PAC'97, Vancouver.

[20] A. Ueda, T. Ushiku, T. Mitsuhashi, "Construction of Travelling Wave Kicker Magnet and Pulse Power Supply for the KEK-Photon Factory Storage Ring", PAC'01, Chicago,
[21]H. Hahn, N. Tsoupas, J.E. Tuozzolo, "The RHIC Injection kicker", PAC'97, Vancouver.

[22] H. Hahn, W. Fischer, V.I. Ptitsyn, and J.E. Tuozzolo, “All-Ferrite RHIC Injection Kicker", PAC'01

[23]H. Hahn, W. Fischer, Y.K. Semertzidis, D.S. Warburton, "UP-Graded RHIC Injection kicker System", PAC'03, Portland.

[24] C.C. Jensen, G.E. Krafczyk, "NuMi Proton Kicker Extraction System", PAC'05.

[25]L. Ducimetière, U. Jansson, G.H. Schröder, E.B. Vossenberg, M.J. Barnes, G.D. Wait, "Design of the Injection Kicker Magnet System for CERN's $14 \mathrm{TeV}$ Proton Collider LHC", Pulsed Power Conf. 1995

[26] R.B. Armenta, M.J. Barnes, E.W. Blackmore, D. Norn, G.D. Wait "Electromagnetic Modelling of the AGS A10 Injection Kicker Magnet”, MT19 2005

[27]L. Ducimetière, G.H. Schröder, J. Uythoven, E. Vossenberg, Q. Han, "CERN Upgrading of the SPS injection Kicker System for LHC Requirements", EPAC'98.

[28] J. Uythoven, G. Arduini, T. Bohl, F. Caspers, E.H. Gaxiola, T. Kroyer, M. Timmins, L. Vos "Beam induced heating of the SPS fast pulsed magnets", EPAC'04.

[29] J. Rümmler, DESY, "DESY III Dump System with one fast Kicker", PAC'95

[30] W. Zhang, L.A. Ahrens, J. Glenn, J. Sandberg, and N. Tsoupas, "Beam Coupling Phenomena in Fast Kicker Systems" PAC'01, Chicago.

[31]F. Caspers, A. Mostacci, and H. Tsutsui, "Impedance Evaluation of the SPS MKE Kicker with Transition Pieces between Tank and kicker Module", CERNSL-2000-071 (AP)

[32]H. Hahn, A. Dunbar, C.I. Pai, R.T. Sanders, N. Tsoupas, J.E. Tuorzzolo, "The RHIC Beam Abort Kicker System", PAC'99, New-York.

[33] J. Rümmler, "Kicker Types for In- and Ejection in the Damping Rings of TESLA", DESY/MIN December 2000.

[34]T. Toyama, K. Ohmi, Y. Shobuda, "Study of Impedances and Instabilities in J-Parc" EPAC'04.

[35] E. Gaxiola, F. Caspers, T. Kroyer, L. Ducimetière, J. Bertin, "Experience with Kicker Beam Coupling Reduction Techniques", PAC'05

[36] G.D. Wait, R. Armenta, M.J. Barnes, E.W. Blackmore, O. Hadary, L. Ahrens, C. J. Gardner, W. Zhang, "Design Concept for AGS Injection Kicker Upgrade of $2 \mathrm{GeV}$ ' PAC'05

[37] J. H. Dieperink, J. Uythoven, "CERN High precision magnetic field measurements of fast pulsed magnets", $12^{\text {th }}$ International Magnet Measurement Workshop, Grenoble, October 2001

[38] T. Ohkawa, Y. Chiba, M. Wakasugi, T. Katayama, "Magnetic Field Measurements of the Kicker Magnet for Muses" EPAC'02, Paris.

[39] W. Pellico, P. Colestock, "Pulsed Magnetic Field Measurement Using a Ferrite Waveguide in a Phase Bridge Circuit" PAC 97. 